\title{
Persistence and Quiescence of Seismicity on Fault Systems
}

\author{
Matt W. Lee ${ }^{1}$, Didier Sornette ${ }^{2}$ and Leon Knopoff ${ }^{1}$ \\ 1 Department of Physics and Astronomy and Institute of Geophysics and Planetary Physics \\ University of California, Los Angeles, CA 90095 \\ ${ }^{2}$ Department of Earth and Space Sciences and Institute of Geophysics and Planetary Physics \\ University of California, Los Angeles, California 90095-1567 \\ and Laboratoire de Physique de la Matière Condensée \\ CNRS and Université de Nice-Sophia Antipolis, Parc Valrose, 06108 Nice, France
}

(May 15, 2018)

\begin{abstract}
We study the statistics of simulated earthquakes in a quasistatic model of two parallel heterogeneous faults within a slowly driven elastic tectonic plate. The probability that one fault remains dormant while the other is active for a time $\Delta t$ following the previous activity shift is proportional to $\Delta t^{-(1+x)}$, a result that is robust in the presence of annealed noise and strength weakening. A mean field theory accounts for the observed dependence of the persistence exponent $x$ as a function of heterogeneity and distance between faults. These results continue to hold if the number of competing faults is increased.
\end{abstract}

02.50.Ey 64.60.Lx 91.30.Dk

Each of the largest earthquakes in recent decades in Southern California has occurred on a different fault of a complex network. In contrast, most efforts at modeling seismicity and its complexity have been directed at studies of self-organization on a single fault. We show quantitatively that coupling between inhomogeneous faults leads to persistent complementary alternation of quiescence and activity between the faults. This is related to the persistence phenomenon discovered in a large variety of systems [1], which specifies how long a relaxing dynamical system remains in a neighborhood of its initial configuration. Our persistence exponent is found to vary as a function of heterogeneity and distance between faults, thus defining a novel universality class.

Competition between faults has been previously observed qualitatively in a quasi-static self-organizing earthquake-fault model [2], and in a dynamic model of the seismicity on two coupled parallel faults [3]. Our present model is a hybrid of these models of interactive faults. As in [2], the driving stress is applied and the stress upon fracture is redistributed quasistatically through an elastic medium of large extent; as in [3], ruptures can occur on either of two predefined coupled, parallel faults, each with variable friction. Our model is a crack version 四 of the 2-D quasistatic earthquake model of a tectonic plate with long range elastic forces [2]. A thin tectonic plate located in the $x-y$ plane undergoes antiplane scalar shear deformation along the $z$-axis. The plate is discretized in $L \times L$ plaquettes of lattice size $a$ that are oriented at 45 degrees with respect to the edges; elastic bonds connect the nodes of the lattice. We impose a slow, uniform velocity $V_{z}$ between the two opposite edges parallel to the $x$-axis. The system is periodic in the $x$-direction. We imbed two linear faults parallel to the $x$-axis; we can consider more than two faults. The faults have finite, spatially variable, rupture strengths; the other parts of the system are assumed to be much stronger and never rupture. To ensure isotropy, we rupture nodes at the intersections of bonds, rather than individual bonds. The system is loaded until the total stress on one fault node reaches a predefined threshold. At this instant, loading is suspended and the corresponding bonds rupture with a complete relaxation of stress followed by a redistribution of stress within the lattice. If additional nodes become critical, the fracture will continue to grow until no further ruptures occur; this defines the end of an event. At this time, the broken bonds are restored to full strength, and a constant, externally derived, irreversible slip is applied to each of the broken bonds to correspond to the force drop associated with the event. Loading now resumes and the process continues. All bonds have the same elastic constant in all simulations.

In contrast to earlier simulations [2, 4], there is no spatially variable quenched disorder on the faults and we instead introduce annealing of the friction by randomizing the thresholds of ruptured nodes after each rupture to the values $B R(x)$ where $R(x)$ is uniformly distributed in the interval $[1-r, 1+r]$, and $B$ is the average value of the force rupture threshold. Physically, the randomization accounts for the fact that a slip associated with an earthquake brings different asperities in contact that were previously separated, thereby changing the local sliding threshold.

Crack models of this type, with relatively homogeneous fracture thresholds, often display runaways [4, 5], which are finite-size events that span the entire length of a fault, and which reset the stress field everywhere along the fault to zero. Runaways arise because the stress enhancement $\sigma \sqrt{L / a}$ at the crack tips of fractures of size $L$ can overcome any heterogeneity bounded by $B(1+r)$ for a sufficiently large crack. Real earthquake fractures have other length scales, and thus do not have force concentrations that become excessively large due to scaling 
by the length of the fracture. We are concerned that our finding of the persistent complementary alternation of quiescence and activity with a power law distribution of time scales might result from the strong overprint of the stress correlations induced by runaways, and thus might be dominated by finite size effects. We have thus extended our model by introducing strength weakening: as soon as the force on a bond reaches $50 \%$ of its initial breaking strength, the force threshold for rupture of a bond is assumed to decrease linearly with time with a decay rate that is twice the loading rate. The results below do not depend on the specific values of these two parameters as long as strength weakening is present at a rate larger than but comparable to the tectonic loading rate, and the threshold for the onset of weakening is sufficiently small. Strength weakening simulates the effect of stress corrosion in fault zones. Weakening causes ruptures to occur earlier, and thus the energy available for prolonged fracture growth is reduced. In our simulations, we have not observed runaways in these conditions.

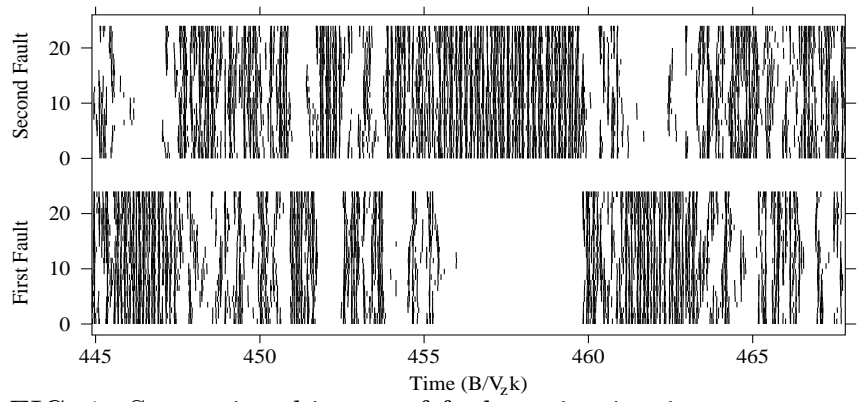

FIG. 1. Space-time history of fault activation in a system of $24 \times 24$ nodes with two faults parallel to the $\mathrm{x}$-axis. We have simulated systems up to $96 \times 96$ with similar results.

Due to the conservative nature of elastic deformation, at least one fault must be active to release the average tectonic loading [2]. In the case of two competing faults, a spontaneous symmetry-breaking occurs, which is an example of a noise-induced transition [6] : there is a welldefined threshold $r_{c}$ for the amplitude of the annealed noise below which all rupture events take place on only one of the two faults and there is no activity on the other fault. The two faults are not decoupled, otherwise they would both be independently active of each other; only their stress fluctuations are decoupled. $r_{c}$ increases with the distance between the two faults. Fig. 1 is an example of the space-time representation of the activity on two competing faults for $r=0.45>r_{c}$. Above $r_{c}$, the symmetry is restored and the rupture events occur on average equally between the two faults.

Shortly after the time that a quiescent fault becomes active, activity on the other may cease along its entire length. The onset of quiescence does not take place instantly, but does so in a finite time after the complementary flip to activity on the other fault. Activity on the quiescent fault is initiated locally at first, and then fractures may appear progressively along its entire length. Occasionally, ruptures on a quiescent fault start to spread, but the effort does not lead to full-fledged activity, as at time 455 on the first fault of the pair. Once an initial seed fracture has been planted on the quiescent fault, it can initiate rupture on its neighbors because of the local high stresses at the edge of the fracture. Opposite these sites, fractures on the active fault are extinguished because they are now in the stress shadow of the newly occurred fractures on the quiescent member. The two faults are now in competition: fractures on the formerly quiescent fault attempt to spread and fractures on the formerly totally active fault, attempt to spread into the "hole" of inactivity at the sites complementary to the 'quiescent' fault. When the two faults are both preoccupied with activity partially distributed along their lengths, it is impossible to decide which had been the active and which the quiescent fault. The formerly quiescent fault may return to a state of quiescence or it may become completely active. Thus there is a finite interval of time between the triggering of activity on the quiescent fault and the extinction of activity on an active fault in the case of a 'flip'. In many cases, there is an outburst of activity, but no flip takes place.

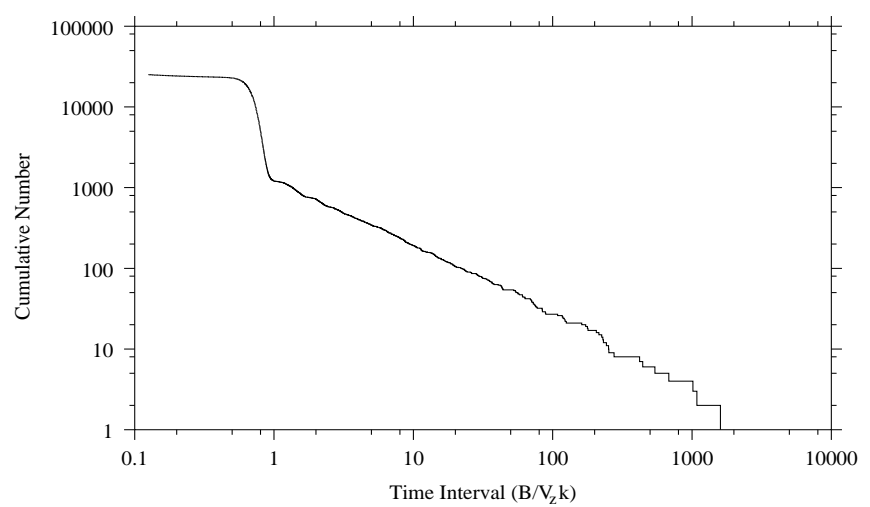

FIG. 2. Cumulative distribution of quiescent time intervals measured in units of $B / V_{z} k$ along one of the faults. The distribution for the other fault is similar.

To define fault activity, we take a moving time average of the total slip in all rupture events on a single fault. A fault is defined to be active when the window-average slip is larger than a given threshold. Fig. 2 shows the cumulative distribution of quiescent time intervals $\Delta t$ along one of the faults. The results are robust with respect to the threshold and size of the window, for large enough $\Delta t$ in comparison with the width of the window. For small $\Delta t$, the distribution is that of the time intervals between two consecutive events on the same fault, which are scaled by the tectonic loading time $B / V_{z} k$, where $k$ is the elastic constant. Greater than this time, the distribution is exclusively that for intervals of flipping of activity between the two faults. The differential distribution of the 
quiescent intervals $\Delta t$ is a power law

$$
P(\Delta t) \sim 1 / \Delta t^{1+x(r, d)},
$$

for all $r>r_{c}(d)$. The larger the value of $r$, the greater the number of flips per unit time. The exponent $x$ thus increases with $r$ (see Fig.3), decreases with increasing fault separation $d$ and is independent of the level of the relaxation threshold and the relaxation rate within the error bars.

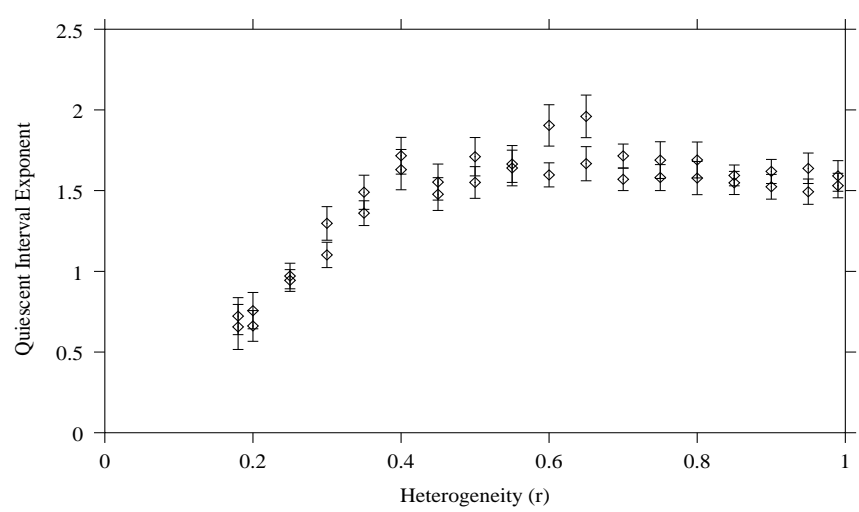

FIG. 3. Dependence of the exponent $x$ on fault heterogeneity $r$ for a fault separation of 0.062 of the fault length. The maximum likelihood exponent is plotted for both faults.

Tectonic loading plays no role in the flipping of a fault from a dormant to an active state since accumulating tectonic stress is continuously released on the active fault. An active fault may flip to quiescence shortly after the time that the dormant fault receives a large enough force from the active one to trigger one site on it into activity. We focus attention on the fluctuations in force on the dormant fault. For long fault lengths, the events that are remotely separated on a single fault are relatively independent. From the central-limit theorem, we expect the distribution of fluctuations in stress on the active fault to be gaussian and this is indeed the case. As the annealing parameter $r$ increases, the increase in the range of rupture thresholds translates to an increase in the variance of the distribution of stresses; the larger the fluctuations in stress on the active fault, the greater the chance of initiating rupture on the inactive fault. For small enough $r$, the activity on a single fault will runaway all too easily. For larger but also low values of $r$, the variance of the stress on the active fault is too small to have significant fluctuations of the stress field to trigger activity on the quiescent fault. This leads to the following prediction: for two faults with the same average threshold $B$ but with different $r$ 's, we expect the more heterogeneous member, that is the one with the largest $r$, to produce greater stress fluctuations on the active fault that might activate the quiescent fault. Therefore, the fault with the greater heterogeneity must be the least active of the two. As a test of this assertion, we find that only fault 2 is active in the case $r_{1}=75 \%$ and $r_{2}=20 \%$. Both faults are active for $r_{1}=75 \%$ and $r_{2}=55 \%$, but fault 2 is active for more than $90 \%$ of the time. As expected, the exponent $x$ decreases with increasing distance between the faults.

The amplitudes of these fluctuations in stress depend on both the length and position of the events on the active fault. Reactivation of the quiescent fault will occur at the rare time that a large fracture occurs on the active fault (but not so large as to cause runaway) so that a large force will be generated near the edge of the crack; the edge of this fracture has about the same $x$-coordinate as the site with low quenched strength on the quiescent fault. Thus, the quiescent fault will become active when a stress fluctuation $\sigma_{a}$ induced by the active fault on it becomes larger than $\left(B_{q}(x)-\sigma_{q}(x, t)\right)_{\text {min }}$, where $\sigma_{q}(x, t)$ is the stress quenched on the quiescent fault. We use a mean field theory to describe the state of stress $\sigma_{a}(x, t)$ due to an active fault on the opposing, dormant fault. Suppose $\sigma_{a}$ fluctuates randomly due to fault activity with the distribution $D_{\sigma_{a}}=C \frac{e^{-\left(\sigma_{a}-\sigma_{0}\right)^{2} / 2 s_{a}^{2}}}{\sqrt{2 \pi} s_{a}}$ for $0 \leq \sigma_{a}<$ $G(d) B(1+r)$. $C$ is the normalizing factor which accounts for the compactness of the distribution support and $G(d)$ is the Green function that transfers stress from the active fault to the quiescent one. $\sigma_{0}$ is the mean stress equal to $B G(d)$. The standard deviation $s_{a}$ must perforce be much less than $\mathrm{Br}$. There is a quenched stress 'barrier' $\Sigma=B_{q}(x)-\sigma_{q}(x)$ on the quiescent fault, where $B_{q}$ and $\sigma_{q}$ are the frozen fracture thresholds and stresses on it, the latter due to the nonuniform slip on it at the time of onset of quiescence. We model the distribution of barriers $\Sigma$ as $D_{\Sigma}(\Sigma)=C^{\prime} \frac{e^{-\left(\Sigma-\Sigma_{0}\right)^{2} / 2 s_{\Sigma}}{ }^{2}}{\sqrt{2 \pi} s_{\Sigma}}$ where $s_{\Sigma} \ll B r$. The variances $s_{a}^{2}$ and $s_{\Sigma}^{2}$ are in general different because they reflect different stress fluctuations : $s_{a}^{2}$ is averaged over all possible stress configurations induced by the active fault on the dormant fault while $s_{\Sigma}^{2}$ is controlled by the variability of the quenched stress left over from the last events before quiescence.

The distribution of quiescent intervals $\Delta t$, for some fixed $\Sigma$, is the same as the distribution of the number (proportional to $\Delta t$ ) of events on the active fault that are necessary to get $\sigma$ larger than some fixed $\Sigma$ :

$$
P_{\Sigma}(\Delta t)=\int_{\Sigma}^{\Sigma_{\max }} d E^{\prime} D_{\sigma}\left(E^{\prime}\right)\left(\int_{0}^{\Sigma} D_{\sigma}\left(E^{\prime}\right) d E^{\prime}\right)^{\Delta t-1} .
$$

The complete distribution of times to reactivation by an isolated event on the quiescent fault is the sum of (2) over all possible values of $\Sigma$ weighted by its corresponding distribution:

$$
P(\Delta t)=\int_{0}^{\Sigma_{\max }} D_{\Sigma}(\Sigma) P_{\Sigma}(\Delta t) d \Sigma
$$

For $\Delta t$ large, (2) and (3) give (1) with

$$
x=\frac{s_{a}^{2}}{s_{\Sigma}^{2}} .
$$


For the Weibull distribution $D_{\Sigma}=e^{-\left(\frac{\Sigma-B}{s_{\Sigma}}\right)^{n}}$, and a similar expression for $D_{\sigma}$ we get $x=s_{a}^{n} / s_{\Sigma}{ }^{n}$. Very long tailed power law distributions for $P(\Sigma)$ gives an exponential distribution for (1).

Since $s_{a}$ is an increasing function of $r$, we expect from (4) that $x$ will also increase with $r$ for smaller values of $r$, as observed (Fig. 3). For $r>0.4$, the fluctuations in stress saturate and $x$ becomes independent of $r$. Also $s_{a}$ is a decreasing function of fault separation $(d)$ due to the smoothing of stress inhomogeneities with distance. Our results are robust whether the breaking strength distribution is power law or uniform, and the distributions need not be the same from fault to fault. Our results are found to hold for up to 10 faults in competition which is the maximum number of faults we have considered.

The number of flips $m(t)$ during a time interval $t$ increases as $m(t) \sim t^{x}$, and hence is a self-affine function of dimension $x<1$ on the time axis. To see this, consider $m$ successive events separated in time by $\Delta t_{i}, i=1, \ldots, m$, where $\Delta t_{1}+\Delta t_{2}+\ldots+\Delta t_{m}=t=m\langle\Delta t\rangle$, and

$$
\langle\Delta t\rangle \sim \int^{\Delta t_{\max }} d t \frac{t}{t^{1+x}} \sim \Delta t_{\max }^{1-x}
$$

Since the maximum $\Delta t_{\max }$ among $m$ trials is typically given by $m \int_{\Delta t_{\max }}^{\infty} \frac{d t^{\prime}}{t^{\prime 1+x}} \sim 1$, we have $\Delta t_{\text {max }} \sim m^{\frac{1}{x}}$. Thus $t=m\langle\Delta t\rangle \sim m^{\frac{1}{x}}$, i.e. $m \sim t^{x}$, which is valid for $x<1$. For $x>1, m \sim t$.

The power law distribution of time intervals between flips implies nonstationarity and aging. Because of the self-similarity embodied in the power-law distributions, we can state that the longer since the last flip of activity, the longer the expected time till the next [7]. In other words, any expectation of a flip that is estimated today depends on the past in a manner which does not decay. This is a hallmark of aging. The mechanism is similar to the "weak breaking of ergodicity" in spin glasses that occurs when the exponent $x$ of the distribution of trapping times in metastable states is less than one [8].

Observations of activity flipping between fault branches on a time scale larger than inter-earthquake times are difficult to document due to the scarcity of reliable historical data. In a well-documented case [9], localized flips in activity have been reported. Temporal clustering of earthquakes on some faults has been identified (see 10 for a summary), but intermittency and fault interactions in the spirit of Fig. 1 have not been observed, which would be needed to establish the relevance of our flipping mechanism.

We have shown that the competition between faults leads to the persistence phenomenon with a power law distribution of the durations of quiescent phases. Our results suggest that complexity in earthquakes and faulting may be a generic outcome of the dynamics of interacting faults. We believe that a broad study of paleoseismicity on many faults is needed to identify intermittency as we have described it.

Acknowledgements: We are grateful to C. Vanneste for help with programming and to the late S.Beanland for calling her work to our attention. Publication no. XXXX Institute of Geophysics and Planetary Physics, University of California, Los Angeles.

[1] S.N. Majumdar and C. Sire, Phys. Rev. Lett. 77, 1420 (1996); S.N. Majumdar et al., ibid 77, 2867 (1996); ibid 77, 3704 (1996); Derrida B., V. Hakim and R. Zeitak, ibid 77, 2871 (1996).

[2] P. Cowie, C. Vanneste and D. Sornette, J. Geophys.Res. 98, 21809 (1993); P. Miltenberger, Sornette, D. and Vanneste, C., Phys. Rev. Lett. 71, 3604 (1993); D. Sornette, P. Miltenberger and C. Vanneste, Pure Appl. Geophys. 142, 491 (1994).

[3] L. Knopoff, Proc. Nat. Acad. Sciences (USA), 93, 3830, (1996).

[4] D. Sornette and C. Vanneste, Nonlinear Processes in Geophysics 3, 1 (1996).

[5] H.-J. Xu and L. Knopoff, Phys. Rev. E 50, 3577 (1994).

[6] W. Horsthemke and R. Lefever, Noise-induced transitions (Springer Verlag, New York, 1984).

[7] D. Sornette and L. Knopoff, Bull. Seismol. Soc. Am. 87, 789 (1997).

[8] J.P. Bouchaud, J. Phys. I France 2, 1705 (1992).

[9] S. Beanland and K.R. Berryman, New Zeal. J. Geol. Geophys. 32, 451 (1989).

[10] A.J. Crone and K.V. Luza, Geol. Soc. Am. Bull. 102, 1 (1990) 